\title{
El problema de las preferencias en la democracia: un acercamiento desde la óptica de la racionalidad acotada
}

\author{
José Manuel Robles Morales \\ Ernesto Ganuza Fernández \\ IESA-CSIC \\ jmrobles@iesa.csic.es \\ eganuza@iesa.csic.es
}

\section{Resumen}

Uno de los principales temas de discusión de la teoría política contemporánea es la cuestión de la naturaleza de las preferencias de los ciudadanos y la capacidad atribuida a estos para participar de forma directa en los asuntos públicos. Sin embargo, reformas legislativas en Europa abogan por implementar nuevos instrumentos destinados a implicar a la ciudadanía en el proceso de deliberación y toma de decisiones en la gestión pública. Desde la teoría económica de la democracia, a menudo se cuestionan los modelos participativos, entre otras cosas, por la imposibilidad que se imputa a tales modelos para permitir una decisión racional. Se supone que el ciudadano se ve afectado por un amplio conjunto de restricciones, como escasez de información, invariabilidad de sus opiniones y creencias o su perfil no técnico, que han servido como argumento para deslegitimar la viabilidad de la participación directa de la ciudadanía. En este trabajo, pretendemos abordar esta problemática desde una óptica distinta. Desde los años sesenta, han florecido distintos modelos de racionalidad (por ejemplo, la racionalidad acotada, de H. Simon), lo cual ha contribuido a dibujar un perfil de ciudadano más flexible y realista que el ofrecido por la racionalidad clásica. Según estos modelos, los sujetos racionales amoldan su decisión a tenor de las características del entorno y de la información disponible, para, de este modo, tomar una decisión eficiente que responda lo mejor posible a las demandas de dicho entorno. Analizando la participación desde esta óptica, postularemos la pertinencia de formas de participación en un espacio público regulado.

Palabras clave: democracia, teoría de la decisión, deliberación, elección racional, racionalidad acotada.

\section{Abstract. The problem of preferences in democracy: a bounded racionality approach}

One of the challenges face contemporary democracies is linked to the individuals preferences. It has been subject of politics theory and reciently differents legislative reforms in Europe have tried to solve the problem through new mechanism involving citizenship directly in public managment. The aim pursued is to create the conditions to citizens participate into policy-making (getting involved in deliberation and decision making proccess). From democracy economics theory is often questioned the participative models, because, it is said, through these models people can not get involved in a rational decision making procces. The citizens are affected by a huge constrains as information scarce, invariabilty of opinions and believes or because of their non proffesional feature. These elements are taken to denied the viability of citizenship direct participation. In this article, we approch 
this controversy from a differnt point of view. New models of rationality (bouded rationality, H. Simon) has appeared during the last decades which challence somo basic Rational choice hypothesis. H. Simon states the rational agents adapt their decisions taking into account enviromental task and the information available in it. We will analyce political participation from the conception of bonded rationality in order to test participation relevance on public regulated scenary.

Key words: democracy, decision theory, deliberation, rational choice, bounded rationality.

\section{Sumario}

1. El problema de las preferencias en democracia

2. Preferencias, elección racional y racionalidad acotada
3. Participación y deliberación

Conclusión

Bibliografía

\section{El problema de las preferencias en democracia}

En el siguiente trabajo, abordamos la democracia utilizando como eje vertebrador de nuestro análisis el problema de las preferencias individuales. Podríamos decir que, desde siempre, la democracia representativa ha sido objeto de innumerables trabajos de investigación. Su crisis, su defensa, su crítica, su desarrollo, han sido cuestiones recurrentes en la literatura política, sociológica y filosófica (Dahl, 1998; Sartori, 1988; Putman, 1993; Habermas, 2000; entre otros). No obstante, en principio, al plantear el problema de la democracia desde las preferencias individuales, abordamos la discusión desde un contexto específico: el de la teoría de la decisión. Primero, partimos, como la teoría de la elección racional, de la consideración de que los individuos tienen preferencias formadas, lo cual presupone pensar el problema de la democracia desde la importancia que adquiere el individuo y su racionalidad; segundo, pensamos la democracia en tanto sistema político que tendrá como objetivo o, al menos, como horizonte argumentativo, la inclusión de todas las preferencias de los individuos (Dahl, 1998). En este sentido, al pensar en la democracia, lo hacemos, al igual que una larga tradición de los expertos en la elección racional (Friedman, 1953), prestando especial atención al problema de la representación de las preferencias de todos los ciudadanos. Estos planteamientos, como decimos, están en la base de la discusión acerca de la naturaleza de las preferencias (y la racionalidad) y acerca de los mecanismos institucionales adecuados para articular políticamente la legítima expresión de las mismas (Sartori, 1988).

En términos generales, la teoría coincide en el hecho que los mecanismos contemplados para garantizar la concurrencia plural de los ciudadanos tienen que tener como referencia a la justicia distributiva (Rawls, 1978; Elster, 1988; Habermas, 2000; Mouffe, 1993; etc.). Así, la generación de decisiones impar- 
ciales o, en su caso, las mejores decisiones para todos los sujetos implicados, se transforma en un objetivo primordial de cualquier sistema democrático. Esto implica, en justicia, un régimen político que descansa en la previa igualdad política de sus ciudadanos (Rawls, 1978). La cuestión sobre cómo se logra un proceso de decisión pública imparcial se convierte, en consecuencia, en un problema que afectará al diseño mismo de la organización política. Así, el problema de la imparcialidad, de una forma más o menos velada, está presente en las discusiones contemporáneas en torno a la política (Rawls, 1978; Kymlicka, 2003; Elster, 1988; Sartori, 1988; Mouffe, 1993; Habermas, 2000; Sanpedro, 2000).

El problema de la imparcialidad nos remite, pensamos, a dos cuestiones. La primera, hace referencia al modo en que las preferencias son generadas, lo cual conlleva reflexionar acerca del estado de las preferencias, su formación y desarrollo, digamos, en términos institucionales, a la formación de la voluntad política. La segunda alude a los arreglos institucionales destinados a que el conjunto de las preferencias estén presentes en los procesos de toma de decisiones públicas. Es decir, nos permite pensar sobre los procedimientos y los mecanismos de organización política. De cómo se conciba la primera cuestión dependerán en gran medida las formas que adoptarán las segundas. Es decir, si consideramos que las preferencias son generadas por el individuo autónomamente, podemos pensar que el problema de las preferencias en el sistema político se convierte en un problema de representatividad (Gargarella, 1998). Si pensamos que las preferencias son generadas de otra manera, en función del entorno, por ejemplo, el problema de las preferencias adquiere otros matices, incluyendo una dimensión deliberativa (Cohen, 1986; Bohman, 1998).

La diferencia es crucial. Para la primera de las alternativas, las preferencias están formadas, es decir, se presupone que la discusión política tendrá siempre lugar alrededor de preferencias dadas (Samuelson, 1947; Little, 1950; Arrow, 1974; Craven, 1992). Aquí sería fácil trasladar a la política el concepto de equilibrio de mercado, destinando todos los esfuerzos a buscar aquella posición que, al igual que en el mercado (óptimo de Pareto), cristalice el equilibrio entre las preferencias de los individuos (Hardin, 1995). Para muchos teóricos, esta "descripción» convierte la posibilidad de la deliberación en una opción marginal o, al menos, plantea a los procesos deliberativos serios problemas que resolver. Por ejemplo, para Przeworski (1998), la política, entendida siempre a partir de individuos racionales, tiene que ver con medios y no con objetivos, lo que hace que la política descanse en argumentaciones técnicas y no normativas. Para él, la política se basa en creencias endógenas vinculadas a posiciones específicas (distribución de ingresos, relación con las instituciones políticas, etc.) que estarían al margen de la deliberación, es decir, las preferencias estarían dadas y ningún proceso deliberativo podría modificarlas. Si hubiera una modificación, ésta sería fruto, en mayor medida, del cambio en las condiciones sociales previas. Por su parte, Johnson (1998) piensa que las argumentaciones son normativas, pero igualmente entiende que los procesos deliberativos difícilmente pueden modificar las preferencias dadas. 
Gargarella (1998) piensa, así, que lo que hace falta al sistema político contemporáneo es incrementar su representatividad y no tanto su dimensión deliberativa ${ }^{1}$.

Sin embargo, si pensamos que las preferencias son generadas de otra manera, es decir, si no son fruto únicamente del individuo y de condiciones estructurales, la cuestión de las preferencias y de la imparcialidad abre las puertas a la inclusión de prácticas deliberativas. Desde esta segunda opción, se considera que el individuo tiene igualmente preferencias racionales. Sin embargo, su formación está estrechamente ligada a un proceso social. Por tanto, al hablar de preferencias, hacemos referencia a un proceso, a una acción, en la que se pueden ver involucrados distintos agentes (humanos, culturales, físicos, etc.). Para nuestro problema, esto significa que hay que considerar el medio junto al cual las preferencias se hacen explícitas. En este sentido, no se puede utilizar el concepto de equilibrio empleado anteriormente, pues cualquier equilibrio es siempre a posteriori, una vez tenga lugar la interacción. Dentro de la filosofía política de la democracia deliberativa, Joshua Cohen (1998), por ejemplo, pretende pensar así en el pluralismo y la deliberación, aunque quizás sea Habermas (2000) quien mejor pueda plantear esta alternativa. Aquí no se cuestiona, en la mayoría de los casos, a las instituciones representativas, bien se entienda por ello a los grupos corporativos o al sistema político, pero sí se abraza una dimensión deliberativa como proceso mediante el cual la política puede (y tendría que) operar (Weber, 1991; Sampedro, 2000). Se entiende que, de otra manera, no se podrían representar, en las decisiones políticas, los intereses del conjunto de la ciudadanía o una parte de ella.

La teoría democrática ha planteado distintos procedimientos de participación política, según se tenga en cuenta una u otra de estas posibilidades. Para la teoría económica de la democracia, la cual parte de la invariabilidad de las preferencias, la representatividad ciertamente esconde un orden social que puede sustituir la participación de la ciudadanía, entre otras cosas, debido a su falta de formación e interés por lo público (Schumpeter, 1984; Sartori, 1988). Para la teoría radical de la democracia (Mouffe, 1993), por el contrario, la representatividad garantiza la participación de toda la ciudadanía. Entre ambas posiciones, existe una distancia cualificada. Mientras los primeros conciben la política como un espacio excluyente (no todos los ciudadanos pueden ser políticos), aunque inclusivo mediante la representatividad, los últimos conciben un espacio directamente inclusivo mediante la representatividad. Para ello, una de las mayores preocupaciones de las teorías de la democracia radical es igualar las condiciones de poder que permiten, a la pluralidad ciudadana, intervenir en la vida pública

1. La defensa de la representatividad como medio para alcanzar el mejor diseño democrático habitualmente descansa en esta argumentación. Incluso teorías de la democracia radical, como la de Chantal Mouffe (1993), se inclinan por esta opción, en el entendido de que son la pluralidad de los grupos corporativamente organizados los que tendrían que ocupar un espacio público diferenciado según las preferencias o los intereses de cada grupo. 
mediante una diferenciación de espacios públicos y grupos corporativos (Sartori, 1999).

Si partimos de la concepción de las preferencias como dependientes y relacionadas con el entorno, tendríamos que reflexionar sobre qué procedimientos deben impulsarse para garantizar la participación política. El ideal de la democracia deliberativa tiene, como criterio de legitimidad, el razonamiento público y libre entre ciudadanos iguales (Cohen, 1986). Esto supone, entre otras cosas, que la orientación de la discusión pública debe estar presentada (razonada) de forma que haga posible la consecución de objetivos comunes sin que, para ello, se tenga que presuponer un consenso previo (Bohman, 1998). De este modo, entendemos que las decisiones y la imparcialidad se fraguan mediante la discusión y la argumentación. Al igual que antes, las derivas prácticas de estas premisas también nos llevan a planteamientos distintos. Por ejemplo, la teoría política weberiana (Weber, 1991) concibe la política asociada a la deliberación parlamentaria. A diferencia de la teoría económica de la democracia, la deliberación presupone un espacio de segundo orden en el que la discusión y las decisiones motivadas racionalmente son observadas como base del procedimiento que mejor garantiza las decisiones imparciales. Esto implica un escenario general que sobrepasa los posicionamientos previos de los individuos y, por tanto, se prima la discusión sobre un interés general y no la negociación entre intereses particulares. Habermas invierte el prisma weberiano y lleva la deliberación a la calle. Así, la deliberación en el espacio público, es decir, la deliberación generalizada entre la ciudadanía, es la que tematizaría los problemas que, posteriormente, deben recoger los representantes políticos. Como vemos, el problema de las preferencias se convierte para Habermas en el problema de la formación comunicativa de la voluntad política. Sampedro (2000), por su parte, al hablar de la formación de la voluntad política, concibe pequeños espacios deliberativos que complementarían la opinión pública generalizada (y representativa) con una opinión pública más cualificada.

En un momento en el que el debate acerca del actual régimen político motiva discusiones y reflexiones, discutir el problema de las preferencias en la democracia persigue comprender la posibilidad de la participación directa como una vía más de organización. Habitualmente, entendemos la democracia contemporánea como expresión de una evolución histórica que hizo inviable el concepto de participación como procedimiento (Dahl, 1998; Sartori, 1988). Dos razones suelen darse, aunque no necesariamente juntas. La primera y fundamental es de orden técnico: la ampliación de los sistemas políticos parejo al desarrollo de los estados nación haría inviable una discusión a gran escala que incluyera al conjunto de la ciudadanía. La segunda, hace referencia al estado de las preferencias. A través de ella se expresa la escasa formación política de la ciudadanía para poder participar, y como consecuencia, se apela a un problema en torno a la calidad de las preferencias.

En este trabajo analizaremos un concepto de racionalidad distinto a aquél que rechaza la argumentación como mediación política. Esta idea de raciona- 
lidad hace un énfasis especial en el carácter adaptativo de las preferencias, así como en la importancia del contexto de decisión en el proceso de formación de las mismas. Este modelo nos permite concebir una organización política en la que la participación deliberativa sea una opción adecuada. En este sentido, como ya expresara también Weber al hablar del parlamentarismo (1991), el estado nación puede albergar procesos deliberativos dentro de un espacio institucionalmente protegido. La cuestión es que si pensamos la racionalidad dentro de un proceso de interacción, la cuestión sobre la cualificación previa de los individuos, es decir, su competencia racional previa, queda relegada a un segundo plano ${ }^{2}$. De este modo, vamos a ver, primero, las implicaciones que se desprenden de una concepción distinta de racionalidad mediante la teoría de la racionalidad acotada, con el objetivo de acercarnos a las implicaciones de un proceso político entendido en términos participativos.

\section{Preferencias, elección racional y racionalidad acotada}

La teoría de la elección racional (TER) bebe de las fuentes de la filosofía de la ilustración, principalmente de la obra de los filósofos John Locke y David Hume. En una de sus más famosas frases, Hume afirma que la razón es la sierva de las pasiones. A través de esta frase, que ha llegado a ser enormemente conocida, Hume pone el acento sobre el hecho de que nuestros actos están, en última instancia, movidos por los deseos de lograr nuestras expectativas y nuestros objetivos, y destaca la escasa trascendencia que tiene la razón en la motivación de nuestras acciones. Si se asume que las pasiones y no la razón son las motivadoras de la acción, poco espacio le resta al observador para determinar las características que mueven a los actores sociales. Es decir, poco se puede determinar sobre la racionalidad de los motivos de las acciones. Regido por este principio, Hume llega a afirmar:

No es contrario a la razón el preferir la destrucción del mundo entero a tener un rasguño en mi dedo [...]. Tampoco es contrario a la razón el preferir un bien pequeño, aunque lo reconozca menor, a otro mayor, y tener una afección más ardiente por el primero que por el segundo. (Hume ${ }^{3}$, Tratado de la naturaleza humana)

No es Hume, ciertamente, el primero que, en la historia del pensamiento occidental, pone de manifiesto la incapacidad de la razón para motivar la acción, sin embargo, nunca antes se había expresado de forma tajante y definitiva. Dada esta premisa, la consecuencia lógica parece ser que la racionalidad deberá predicarse, más que de los objetivos que persiguen los agentes, de cómo gestionan éstos los medios que tienen a su disposición para lograrlos.

2. Veáse la interesante argumentación de Max Weber al respecto (1991).

3. Para esta cita del Tratado de la Naturaleza Humana, de D. Hume, hemos utilizado la edición de la Editorial Tecnos de 1988. 
Dado que los deseos, y no la razón, son los únicos motivadores de la acción, el espacio de aquélla deberá reducirse al manejo de los instrumentos que posibiliten la consecución satisfactoria de los objetivos. De esta forma, los deseos, las preferencias a partir de ahora, serán concebidos como los motivadores de la acción sin que cuenten, de forma justificada, como variable de peso en la explicación de la racionalidad humana.

La TER, como hemos mencionado, es heredera de esta concepción. En la jerga académica, se suele afirmar que las preferencias "son dadas", con lo que se hace referencia al hecho de que presumimos que los agentes poseen algún tipo de preferencia, pero que no se hará referencia a su cualidad, género o certeza, sino, simplemente, a su existencia. Lo que conocemos de las preferencias, según la TER, es a través de las acciones de los agentes. Esta relación ha sido tomada, en origen, del denominado Criterio de Condorcet (Craven, 1992). Según este criterio, se puede identificar formalmente preferencia y elección si la persona que decide elige su alternativa preferida (Craven, 1992). El supuesto parte de que lo contrario, es decir, preferir una alternativa y elegir otra es una irracionalidad, por lo que, al elegir un curso de acción, estamos desvelando nuestras preferencias (preferencia revelada).

Muy relacionado con lo que se ha mencionado anteriormente, diremos que otra de las fuentes clásicas de la TER es el utilitarismo. De la obra de J. S. Mill y, especialmente, de J. Bentham, la teoría toma otro de sus fundamentos básicos, a saber, el carácter autointeresado de la acción. Los agentes racionales preferirán un determinado curso de acción sobre otro en la medida en que le proporcione un mayor placer o le evite mayor dolor. Esto supone una matización sobre el supuesto humano de las pasiones. En palabras de Mill: «[...] el credo que acepta la utilidad o el principio de la mayor felicidad [...] sostiene que las acciones son justas en la medida en que tienden a promover la felicidad e injustas en cuanto tienden a producir lo contrario de la felicidad» (Mill, 1971: 141). La relación entre preferencia y utilidad es, de este modo, extremadamente simple. El denominado concepto de utilidad de Bentham (1996) establece que las acciones más deseables aportan más utilidad que las menos deseables o, en otras palabras, «[...] si una persona prefiere la situación $a$ a la situación $b$, diremos que la utilidad asignada a la opción $a$ es mayor que la asignada a la opción $b_{»}$ (Nicholson, 2002). Este principio representa el soporte cognitivo de la teoría. Sin embargo, en la versión clásica de la teoría o en su thin version (Elster, 1986), poco más se dice en este sentido. Así, cuestiones como las causas, la racionalidad o la viabilidad de las preferencias son tomadas como dadas, mientras que la racionalidad de las acciones se predicará, principalmente, de la correcta disposición y manejo de los medios para alcanzar los fines ${ }^{4}$.

4. Las razones esgrimidas para sostener esta postura teórica son muchas. En primer lugar, las preferencias y todas las variables en torno a ella son tomadas como dadas, es decir, como cláusulas ceteris paribus, con la idea de controlar mejor las variables operativas que le interesan a la teoría. Del mismo modo, acotar las motivaciones posibles para la acción permite 
De este modo, el papel que les resta a las preferencias en la teoría es, así, eminentemente mecánico. En este sentido, la teoría explicita que las preferencias deben cumplir una serie de requisitos: completitud, transitividad y continuidad o extensionalidad (Craven, 1992). El primero de ellos sostiene que todo agente que se enfrente a dos opciones cualesquiera, $\mathrm{A}$ y $\mathrm{B}$, debe especificar: 1) si A es preferida a B; 2) si B es preferida a A, o 3) si A y B son igualmente atractivas (criterio de indiferencia). La transitividad ofrece consistencia para elecciones en las que los agentes deben elegir entre más de dos cursos de acción. De esta forma, y apoyándose en la cláusula anterior, la teoría explica que si $\mathrm{A}$ es preferida a $\mathrm{B}$ y $\mathrm{B}$ es preferida a $\mathrm{Z}$, A deberá preferirse a $\mathrm{Z}$ (Craven, 1992). Por último, la continuidad o extensionalidad especifica que si $A$ es preferida a $\mathrm{B}$, las situaciones suficientemente «cercanas» a A deberán preferirse a B. En otras palabras, cualquier par de situaciones percibidas por el agente como descripciones alternativas del mismo problema llevarán a éste a preferir la misma opción (Arrow, 1974). Este último supuesto está concebido con la idea de permitir analizar respuestas de los individuos a cambios relativamente pequeños en el ambiente. Del correcto ajuste a estos principios operativos en la consecución de los objetivos perseguidos, se predicará la racionalidad de una acción.

No han sido pocas las críticas que se han vertido sobre esta forma de definir el comportamiento racional (Gigerenzer y Selten, 2002). Sin lugar a dudas, no es éste el momento para hacerse eco de todas ellas, sin embargo, sí nos detendremos en una que interesa especialmente, dados los objetivos de este trabajo: las referentes al realismo del criterio de extensionalidad. Tverky y Khaneman (2000) dieron cuenta del carácter contrafáctico de este criterio al analizar en qué forma el modo en el que está enmarcado un determinado entorno de decisión determina las preferencias de los agentes. En uno de los múltiples casos citados por Tversky y Khaneman se analizaban las preferencias de un grupo de en torno a quinientos individuos sobre el tratamiento médico contra el cáncer, en especial, de la radioterapia. A un subconjunto de estos individuos se les presentó información sobre el porcentaje de enfermos de cáncer que, tras ser sometidos a radioterapia, lograron superar la enfermedad. Por el contrario, a un segundo grupo se les daba la misma explicación presentando información sobre las personas que, pese a haberse sometido al tratamiento, no lograron superar su enfermedad. El resultado mostró hasta qué punto el modo en el que la situación es enmarcada (personas curadas en el primer caso contra personas fallecidas en el segundo caso) influye en las preferencias de los agentes. Más de un $44 \%$ de las personas del primer grupo se mostraron favorables a este tipo de tratamientos, mientras que sólo el 18\% del segundo grupo expresaron esta misma opinión.

controlar la proliferación de explicaciones causales ad hoc que mermen la capacidad explicativa de la teoría. Sin embargo, tal y como dice Rescher (1993) con esta postura, la teoría cae en la falacia de confundir un aspecto de la racionalidad, el informativo, con el conjunto de mecanismos que explican el comportamiento racional entre los que figura, como parte fundamental, la racionalidad de los motivos para la acción. 
También son importantes las críticas a uno de los supuestos auxiliares del criterio de extensionalidad, el conocido como propiedad Alpha (Sen, 1986). Esta propiedad mantiene que si se elige el objeto $x$ dentro del conjunto $S$ pero $x$ también pertenece al subconjunto $T$ del conjunto $S$, entonces $x$ deberá ser también elegido en $T$. En otras palabras, cualquier acción realizada de la siguiente forma violaría dicha propiedad:

i) $X$ es elegido dadas las opciones $X$ e $Y$.

ii) $Y$ es elegido dada las opciones $X, Y$ y $Z$.

Esta propiedad mantiene, al igual que el criterio de extensionalidad, que, dados dos entornos que se planeen de la misma forma, la opción elegida deberá ser la misma. En el caso del ejemplo anterior, la diferencia entre el entorno 1) y 2) es la inclusión de una nueva opción, a saber, la opción $Z$. En principio, esto licitaría al agente a elegir $Z$ sobre la opción escogida con anterioridad, pero nunca la opción presente en el entorno anterior, la cual no fue elegida. Sin embargo, son múltiples los casos en los que se viola este criterio. Gigerenzer (2000) presenta, en Adaptive Thinking, algunos ejemplos. Uno de ellos se resume de la siguiente forma:

En una cena, se pasa, entre los asistentes, una bandeja con fruta. Cuando la bandeja llega a Mr. Polite sólo queda una pieza de fruta en la bandeja. Si Mr. Polite hubiera estado cenando solo, la hubiera cogido sin más; no hubiera habido ningún dilema. Sin embargo, estando en compañía, él debe elegir entre la manzana $(Y)$ o nada $(X)$. Dado que es una persona muy educada, decide optar por $X$ y no tomar la última pieza de fruta de la bandeja. Si la bandeja hubiera contenido otra pieza de fruta $(Z)$, él podría haber escogido $Y$ sobre $X$ y $Z$ sin tener que violar los estándares de buena conducta. Se trata de un caso en el que se viola claramente la propiedad Alpha, debido a que la opción elegida en el segundo entorno es precisamente la que se rechazó en el primero. De este modo (se puede pensar que), el carácter social del entorno no es marginal respecto a las preferencias que revelan los agentes. Parece, dadas estas razones, que, entre dos situaciones en las que el agente se enfrente a la misma decisión, la elección de la misma opción no está garantizada.

Estas dos críticas al criterio de extensionalidad motivan serias dudas sobre su recurrencia en entornos reales. Sin embargo, un área en la que se ha indagado menos las dificultades que entraña este criterio es en la de la acción colectiva. Desde este ángulo, es interesante preguntarse en qué medida afectan las críticas al concepto de extensionalidad a la acción individual en entornos de interacción. Nos gustaría adentrarnos en este tema partiendo de la relectura que realiza Sen (1986) del conocido dilema del prisionero. El dilema del prisionero ofrece la siguiente estructura:

Dos ladrones son atrapados y acusados de perpetrar un crimen para el que la policía no cuenta con suficientes pistas. Ambos ladrones son encerrados en celdas distintas e incomunicados. Se les ofrece la posibilidad de que confiesen su crimen bajo la promesa de que se les reducirá la condena. 
Así, si ambos confiesan, la reducción será de la mitad de lo prescrito para el delito del que se les acusa (veinte años), por lo que estarán en la cárcel un total de diez años. Si uno de los dos transige y confiesa mientras que el otro mantiene la boca cerrada, éste último será condenado a la pena máxima en base a las pruebas ofrecidas por el delator, mientras que éste quedará en libertad. Si ninguno de los dos confiesa, serán acusados de un delito menor y pasarán en la cárcel sólo dos años. Expuesto en forma de tabla, quedaría de la siguiente forma:

Dilema del prisionero ${ }^{5}$

\begin{tabular}{|l|l|l|l|}
\cline { 3 - 4 } \multicolumn{2}{|c|}{} & Prisionero 2 & \multicolumn{1}{|l}{} \\
\cline { 3 - 4 } \multicolumn{2}{|c|}{} & Confiesa & No confiesa \\
\hline Prisionero 1 & Confiesa & $-10,-10$ & $0,-20$ \\
\hline & No confiesa & $-20,0$ & $-2,-2$ \\
\cline { 3 - 4 } & &
\end{tabular}

En principio, existe una estrategia dominante para cada prisionero. Esta estrategia consiste en confesar sin importar lo que haga el otro prisionero. Esto es así debido a que el prisionero 1 pensará que si el prisionero 2 confiesa, confesando también él, su castigo será menor, es decir, en vez de veinte años le caerán diez años. Por otra parte, si el prisionero 2 no confiesa, para él es mejor confesar, ya que en ese caso se libraría de toda condena. Este mismo razonamiento es el que llevará al prisionero 2 a confesar. De ser así, la teoría predice que ambos confesarán y, por lo tanto, estarán en la cárcel un periodo de diez años. Esto significa, y por esta razón hablamos de dilema, que, siguiendo lo expresado por la teoría, los individuos alcanzarán un equilibrio que no es el óptimo, pues si los dos se hubieran mantenido sin confesar, el castigo hubiera sido menor.

Pese a que no cabe duda de que formalmente este razonamiento es impecable, en la práctica se producen un amplio conjunto de situaciones muy similares que no terminan en resultados subóptimos. Sen dice lo siguiente, al respecto de este asunto:

En particular, en la historia del dilema del prisionero, el bien general ${ }^{6}$ puede interpretarse como una regla de no confesión, la cual es beneficiosa para ambos y el vehículo para su consecución será un tratado mutuo de no confesión. Si un tratado de ese tipo puede ser alcanzado o impuesto, los dos prisioneros obtendrán un resultado mejor. (Sen, 1986: 70)

5. Esquema del dilema del prisionero según la explicación de Sen en Behaviour and the concept of preference (1986).

6. Sen se refiere, por ejemplo, a objetivos comunes, tales como los descritos por Rousseau bajo el nombre de contrato social. 
De hecho, Sen mantiene que no es necesario recurrir a la existencia de un contrato social para evitar el resultado del dilema del prisionero. En muchos casos, las reglas normativas, morales o religiosas pueden desempeñar el papel atribuido a dicho contrato. Así, «supón que cada prisionero del dilema actúa [...] siguiendo el dictado de no defraudar al otro sin tener en cuenta las consecuencias para sí mismo" (Sen, 1986: 70). Lo que se desprende del enfoque de Sen del dilema del prisionero es que: 1) en una decisión que implique determinados tipos de deliberación colectiva, el respeto de axiomas de la TER, tales como la extensionalidad o las creencias egoístas, pueden conllevar un resultado subóptimo, y 2), en la práctica, esta circunstancia no siempre se produce, debido a la existencia de mecanismos sociales que facilitan que se produzcan equilibrios óptimos.

Sin embargo, existe una cuestión más de fondo relacionada con las preferencias. El carácter preestablecido de determinados axiomas de la TER lleva consigo que apenas exista cuestionamiento de los mismos. No son pocas las revisiones sobre el carácter fundamental del egoísmo en las acciones individuales y no son pocos los que han tratado de ofrecer alternativas más flexibles que hagan de las preferencias un elemento más realista (Simon, 1983). Sin embargo, se ha transigido menos en relación con el carácter adaptativo de las preferencias. Tanto es así, que es uno de los elementos que separa la TER de su más serio competidor en la descripción del comportamiento racional, la teoría de la racionalidad acotada (TRA). A diferencia de la TER, la TRA parte de una concepción ecológica del sujeto, según la cual la racionalidad del agente se predica de su capacidad para tomar buenas decisiones sacando el mayor partido posible a la estructura del entorno (Todd y Gigerenzer, 2003).

La TRA comparte con la TER el supuesto metodológico de que las restricciones presentes en el entorno de decisión determinan sobremanera las decisiones de los agentes. Sin embargo, se distancia de esta teoría en lo que respecta a la descripción de las características cognitivas de los agentes, así como en la forma en la que éstos se «relacionan» con dicho entorno restringido. La TRA parte de la existencia de restricciones, tanto contextuales (costes asociados a la búsqueda de la información o tiempo de búsqueda), como cognitivas (costes asociados al manejo, a la evaluación o a la comparación de las opciones de decisión). Sin embargo, a diferencia de la TER, estas restricciones no representan una desviación del entorno «canónico» de decisión, que estaría representado por un entorno de información completa, sino una característica realista de las decisiones a las que se enfrentan los agentes (Selten, 2002). Dada esta postura, la TRA analiza la forma en la que, partiendo de estas restricciones, los agentes son capaces de obtener resultados satisfactorios. La racionalidad es, así, una cuestión de interacción con el entorno y no de la consecución óptima de los objetivos individuales autointeresados.

Esto significa varias cosas. En primer lugar, que en la TRA el agente social es un sujeto capaz de adaptarse a las circunstancias y que, en segundo lugar, esta capacidad adaptativa también alcanza a sus preferencias. Una de las teorías más sugerentes que abordan este tema es la conocida como teoría de la adap- 
tación aspirada (Selten, 1998). Según esta teoría, el modo como alcanzamos las preferencias que consideramos satisfactorias es el resultado de un proceso escalonado de búsqueda. En este proceso escalonado existen distintos niveles a los cuales se aspira en función de la dificultad del contexto ${ }^{7}$ en el que se tomará la decisión. Muy grosso modo, según Selten, en nuestra adaptación al entorno iremos avanzando en niveles de exactitud de nuestras aspiraciones y, por tanto, de nuestras preferencias, según el nivel de exigencia que plantea cada uno de los contextos. Según esto, la forma en la que procederíamos para tomar una decisión que desemboque en una acción, es ajustando nuestras capacidades, nuestras preferencias y las características del entorno.

Esta idea general puede sintetizarse a través de la metáfora que utilizó H. Simon (1983) para ilustrar el sentido que tiene la racionalidad acotada. Según esta metáfora, el comportamiento racional puede entenderse como un par de tijeras. Una de las hojas de estas tijeras se correspondería con la estructura del ambiente en el que se va a tomar la decisión, mientras que la otra correspondería a las capacidades y preferencias del sujeto. Así, para comprender el comportamiento de éste, debemos entender la acción como si las dos hojas de esta tijera tuvieran que cerrarse de forma que las dos estructuras, mental y contextual, se encajaran. De este modo, y mientras que la estructura del ambiente es más o menos previsible ${ }^{8}$, tanto las capacidades cognitivas como las preferencias del agente pueden adaptarse para poder explotar las estructuras particulares del ambiente. Para facilitar este «ajuste», un sujeto racional pondrá en juego un conjunto de herramientas ${ }^{9}$ que le permitirán sacar el mayor partido posible al ambiente en el que tiene que tomar su decisión.

Partir de esta descripción de los agentes sociales nos posibilita matizar la solución que ofrece Sen (1986) al dilema del prisionero. En definitiva, se trata de una concepción de las preferencias del sujeto que pone de manifiesto su

7. Es decir, los objetivos de los agentes son más «modestos» en función de las dificultades (restricciones) presentes en el contexto de decisión.

8. La previsibilidad del contexto no descansa en condiciones inmutables del entorno, sino en una característica propiamente racional, según la cual se reglamentan, jurídica y no jurídicamente, diferentes tipos de acción. Es esa previsibilidad la que, según Weber (y más tarde Coleman o Habermas), facilita el derecho, pero es también la que facilita cualquier institución creada o heredada. Tal previsibilidad permitiría a los diferentes agentes actuar racionalmente de un modo no necesariamente autointeresado (Habermas, 1988), pero más que nada nos dice la importancia que adquiere el contexto para el proceso de toma de decisiones. El contexto está abierto al cambio social, la peculiaridad de las sociedades contemporáneas puede ser caracterizada, entendiendo tal contexto de forma creada y dependiente de la propia acción entre los individuos.

9. Este tipo de herramientas son conocidas en la TRA como heurísticos. «Genéricamente, los estudiosos de la racionalidad acotada, han denominado heurísticos a los algoritmos (conjunto finito de operaciones que nos permiten resolver un determinado problema) utilizados para salvar nuestras restricciones cognitivas. Los heurísticos son atajos cognitivos que posibilitan a los individuos realizar evaluaciones sobre la base de una o varias reglas o estructuras básicas, evitando así los costes relativos a la exploración exhaustiva de un conjunto amplio y complejo de posibilidades y, al mismo tiempo, adaptándose a las circunstancias que el ambiente le plantea» (Robles, 2005). 
carácter adaptativo. Bajo esta descripción, los agentes son capaces de flexibilizar sus preferencias a tenor de determinadas variables de tipo contextual presentes en el entorno de decisión. Según la explicación de Sen, estas variables eran la concurrencia de determinados preceptos morales o religiosos o la existencia de un acuerdo social. Esta circunstancia determina que el tipo de preferencias que exhiben los agentes sean distintas de las descritas por la TER. Esto significa que las preferencias no están cerradas de antemano, sino que pueden modificarse en función de las características y las posibilidades que ofrece el contexto. En el caso que nos ocupa, esto significa que al actuar o, si se quiere, al participar en un entorno determinado, el agente conforma sus preferencias adaptándolas a las características constitutivas del entorno. Así, el hecho de estar sometido a un entorno de deliberación común hace que, tal y como muestra el dilema del prisionero, un comportamiento autointeresado obtenga un resultado subóptimo ${ }^{10}$. Esto no significa que la descripción rechace por completo el carácter autointeresado de la elección individual, sino que, simplemente, reserva su capacidad explicativa para determinado tipo de entornos de decisión. Sin embargo, sí pone en cuestión el carácter extensional de las preferencias. Es decir, los agentes en los entornos reales de decisión adaptan sus preferencias con el objetivo de alcanzar mejores resultados o con el objetivo de facilitarles la consecución de los mismos. Este acuerdo sólo es posible si se adaptan sus preferencias a dicho entorno. Un supuesto del que carece la teoría de la elección racional.

\section{Participación y deliberación}

Uno de los principales argumentos defendidos por la teoría de la racionalidad acotada es el carácter contextual de la decisión. Como hemos dicho, según la TRA, las preferencias individuales se actualizan junto al medio en el que el agente se posiciona, es decir, podemos esperar que un individuo racional tome sus decisiones considerando, junto con sus propias preferencias, las características, las leyes o las normas del medio con el que está interaccionando ${ }^{11}$. Esta propuesta no hace más que acentuar la importancia de la variable contextual en el proceso de decisión pública. En nuestra opinión, si el contexto es relevante racionalmente, las posibilidades que abre el entorno deliberativo serán de gran importancia para la formación de las opiniones políticas.

En esta línea se expresa Sen (2000: 153) al mantener que esta concepción de la democracia (los derechos y el fomento a la discusión pública) empuja a

10. En este trabajo, se mostrarán ejemplos variados de acciones deliberativas en las que los agentes muestran preferencias flexibles que permiten alcanzar resultados que no serían posibles según el comportamiento expuesto por la TER.

11. La posibilidad de que el individuo emprenda una acción egoísta es, no obstante, indiferente a nuestro problema. Como decíamos antes, entendemos que es una posibilidad del comportamiento del individuo, pero no la única. 
los agentes sociales a realizar elecciones reflexivas (reflected choices), de manera que el proceso deliberativo resulta crucial en la formación de valores y preferencias sociales. Amartya Sen lleva el debate incluso hacia la concepción de las necesidades, las cuales, más que responder a cualidades inherentes del ser humano, son consecuencia también del entorno social al que pertenece. La deliberación pública, en este sentido, no sólo induce a los distintos gobiernos a hacerse responsables de las necesidades de la población, sino que también es central en la conceptualización de las mismas necesidades (ibídem: 154). En un momento histórico donde la sociología, independientemente de sus interpretaciones y configuraciones, cuestiona en general la posibilidad de una autoridad política imperativa, la adecuación de un entorno político deliberativo adquiere una importancia mayor.

Dado el papel central del entorno de decisión para la consecución de decisiones racionalmente motivadas, en lo que sigue, trataremos de exponer las reglas y los procedimientos que ofrece la democracia deliberativa para promover el acuerdo racional entre ciudadanos ${ }^{12}$. En este sentido, al hablar de un entorno deliberativo, haremos mención explícita a las reglas, las prácticas y las convenciones deliberativas, a saber, un conjunto de elementos que configurarán el contexto político y el proceso de toma de decisiones de forma deliberativa. Así, no hablamos de la deliberación en algún sentido ideal o moral, sino a partir de sus aspectos, si acaso, instrumentales.

En la actualidad, hay ejemplos contemporáneos suficientes para debatir y valorar diferentes tipos de procedimientos deliberativos. Todos ellos se elaboran pensando en un proceso con más o menos participación de la ciudadanía. Es igualmente notoria la inclinación de las administraciones contemporáneas a favorecer la creación de espacios deliberativos, con desigual intensidad, pero que han llegado a reflejarse en cambios legislativos en muchos países europeos (Ganuza, 2004). Igualmente, instituciones internacionales estimulan o recomiendan su desarrollo (OCDE, 2001; CMCE, 2001; PNUD, 2002). Las distintas administraciones territoriales en Europa han desarrollado, durante los

12. La teoría deliberativa ha estado sujeta a un importante conjunto de críticas. Ciertamente, la mayoría de las críticas planteadas a los procesos deliberativos no ponen en cuestión el principal supuesto de la deliberación, a saber, la promoción de acuerdos racionalmente motivados entre los agentes (Habermas, Gutmann y Thompson, Cohen, etc.). Sin embargo, sí destacan la dificultad de asumir, en la vida práctica y cotidiana, algunos de sus supuestos. En primer lugar, se señala la dificultad que entraña la consecución de acuerdos racionales (supuestamente «buenos»). Es decir, no se duda de la bondad del modelo, pero sí de que todos los ciudadanos puedan per se alcanzar buenos razonamientos (Sartori, 1988). En segundo lugar, se entiende que no es posible articular la política contemporánea deliberativamente en grandes extensiones de población, dada la imposibilidad de que pueda participar toda la población y se pueda incluir la multiplicidad de preferencias en un proceso deliberativo (Dahl, Sartori). Sin embargo, en este trabajo, nuestro objetivo no se centra en la capacidad de la deliberación para alcanzar resultados políticamente convenientes. Tampoco nos centramos en las formas de institucionalización de las prácticas deliberativas. Nuestro objetivo es analizar las potencialidades de los entornos deliberativos para la consecución de decisiones racionales. 
últimos años, diferentes experiencias participativas y deliberativas de complejidad variada y a escala diferente (desde un municipio, grande o pequeño, hasta una región o un estado). Sólo mencionaremos, por ejemplo, los jurados ciudadanos en Alemania, Reino Unido, EE.UU. o España; las conferencias de consenso en Dinamarca o EE.UU., o los presupuestos participativos en Brasil, España, Italia o Alemania ${ }^{13}$.

Las experiencias desarrolladas tienen como objetivo la participación de la ciudadanía en un debate público sobre una cuestión concreta, que, en la mayoría de los casos, está vinculada a un proceso de toma de decisiones político. Aquí la discusión no versa sobre los asuntos privados, ni sobre las preferencias particulares, sino sobre una cuestión que afecta al conjunto de la población. Así, el elemento central en el debate es la resolución práctica de las cuestiones propuestas para la discusión. Por lo tanto, la cuestión central no serían las preferencias de los individuos, las cuales no se cuestionan ni se problematizan, sino los procedimientos seguidos para lograr ese entorno deliberativo.

Las experiencias desarrolladas descansan en un conjunto de procedimientos definidos previamente. Pese a su heterogeneidad, todos estos mecanismos presuponen el apoyo de la administración mediante la difusión de la información necesaria para la participación y la pautas y normas de regulación de las mismas (Ganuza, 2005). Esto implica que no todo es válido y que la decisión se fragua en un proceso reglado. En el cuadro 1, reflejamos las características generales de los procedimientos empleados por cada una de las tres experiencias participativas citadas anteriormente.

En cualquiera de los procesos participativos mencionados, el individuo es invitado a una situación pública de argumentación. Su posición sobre la cuestión abordada es sometida a un proceso deliberativo donde tiene que escuchar o, al menos, tiene que respetar la argumentación de las preferencias de los otros. Una vez expuestas las consideraciones particulares, se debate mediante distintos procedimientos qué decisión se adoptará. En el caso de los jurados ciudadanos, el debate tiene lugar durante un tiempo previamente delimitado y la decisión se adopta apelando a la mayoría. En las conferencias de consenso, el debate persigue alcanzar un acuerdo común sobre mínimos. Mientras, en los presupuestos participativos entran en juego varios procedimientos. Por un lado, en la mayoría de ellos suelen debatirse las propuestas en asamblea pública. Por otro lado, suele utilizarse una evaluación técnica con el objeto de ceñir el marco de discusión a las decisiones que son competencia de la administración. Por último, las decisiones finales son, en las experiencias en España y Brasil, alcanzadas mediante criterios redistributivos previamente debatidos por la ciudadanía.

Las reglas generan, en estos procesos, un contexto deliberativo en el que los participantes discuten públicamente. Por lo tanto, los procedimientos son

13. Puede verse una relación de procesos participativos y deliberativos en los países de la OCDE en OCDE (2001), en Sintomer (2004), Ganuza (2004), Font (2001). 
Cuadro 1. Características procedimentales de procesos deliberativos y participativos.

\begin{tabular}{|c|c|c|c|}
\hline & $\begin{array}{l}\text { Jurados } \\
\text { ciudadanos }\end{array}$ & $\begin{array}{l}\text { Conferencias } \\
\text { de consenso }\end{array}$ & $\begin{array}{l}\text { Presupuestos } \\
\text { participativos }\end{array}$ \\
\hline $\begin{array}{l}\text { Participación } \\
\text { (quién participa) }\end{array}$ & $\begin{array}{l}\text { Ciudadanos elegidos } \\
\text { por sorteo según } \\
\text { censo o padrón. } \\
\text { También pueden } \\
\text { participar asociaciones } \\
\text { invitadas. }\end{array}$ & $\begin{array}{l}\text { Ciudadanos previamente } \\
\text { motivados (invitación } \\
\text { a través de medios } \\
\text { de comunicación). }\end{array}$ & $\begin{array}{l}\text { Ciudadanos previamente } \\
\text { motivados y asociaciones } \\
\text { (invitación a través } \\
\text { de medios de } \\
\text { comunicación, cartelería } \\
\text { y otros medios). }\end{array}$ \\
\hline $\begin{array}{l}\text { Delimitación } \\
\text { de agenda (sobre } \\
\text { qué se participa) }\end{array}$ & Administración. & $\begin{array}{l}\text { Administración. } \\
\text { Administración. }\end{array}$ & $\begin{array}{l}\text { Administración y, } \\
\text { en algunos casos, } \\
\text { la ciudadanía. }\end{array}$ \\
\hline $\begin{array}{l}\text { Reglas (cómo } \\
\text { se participa) }\end{array}$ & $\begin{array}{l}\text { Reglas decididas } \\
\text { por la Administración. }\end{array}$ & $\begin{array}{l}\text { Reglas decididas } \\
\text { por la Administración. }\end{array}$ & $\begin{array}{l}\text { Reglas decididas } \\
\text { por la Administración } \\
\text { o codecididas entre la } \\
\text { ciudadanía y la } \\
\text { Administración. }\end{array}$ \\
\hline Información & $\begin{array}{l}\text { Exposición } \\
\text { de distintos expertos. }\end{array}$ & $\begin{array}{l}\text { Exposición de distintos } \\
\text { expertos. }\end{array}$ & $\begin{array}{l}\text { Información difundida } \\
\text { por la Administración. }\end{array}$ \\
\hline Deliberación & $\begin{array}{l}\text { Diálogo con expertos } \\
\text { y entre participantes. }\end{array}$ & $\begin{array}{l}\text { Diálogo con expertos } \\
\text { y entre participantes. }\end{array}$ & Diálogo entre participantes. \\
\hline Decisión & Mayoría. & Consenso. & $\begin{array}{l}\text { Mayoría y, en algunos casos, } \\
\text { criterios de justicia } \\
\text { distributiva (como en } \\
\text { España o Brasil). }\end{array}$ \\
\hline Ejecución & Administración. & Administración. & $\begin{array}{l}\text { Administración. } \\
\text { En algunos casos, se realiza } \\
\text { un control y un } \\
\text { seguimiento ciudadano. }\end{array}$ \\
\hline
\end{tabular}

Fuente: elaboración propia.

públicos y transparentes. Es cierto que, para deliberar, es necesario también información, algo que muchas veces es interpretado como una debilidad de los procesos deliberativos. Sin embargo, al margen de los procedimientos y de los recursos habilitados para dar información, que siempre pueden ser considerados escasos o limitados, hay que valorar la importancia que tiene un debate público como generador de información (para entender el debate, para participar en él), fruto del intercambio de argumentos, etc. Frey, Kucher y Stutzer (1999) mostraron como, en los cantones suizos con una legislación más abierta y proclive a la realización de referéndums, la ciudadanía estaba más informada sobre las cuestiones gubernamentales. No obstante, ningún proceso deliberativo presupone que el individuo tenga conocimientos exhaustivos a priori acerca de lo que se debate. Aun así, la administración invierte 
importantes recursos para lograr que los participantes tengan a su disposición la información necesaria para la participación. Sin embargo, lo que consideramos más importante es la capacidad de un entorno deliberativo para generar información y argumentos para el debate, contribuyendo a su difusión precisamente como algo propio del proceso deliberativo.

En este sentido, un contexto deliberativo facilita la consecución de acuerdos. Esto no significa que el propio hecho de deliberar garantice la resolución de cualquier tipo de conflicto, sino que las decisiones descansan en un procedimiento público, casi siempre revisable, que permite establecer una relación racional entre los participantes. En este sentido, la deliberación presupone, al menos en los procedimientos contemporáneos, un conjunto de reglas que hacen posible la discusión, es decir, se pueden seguir las diferentes etapas por las que se alcanza una decisión. Todas ellas son públicas. En estos casos pensamos, con Subirats (2003), que un individuo puede estar de acuerdo con la decisión final teniendo preferencias distintas. Ha podido escuchar otros argumentos, exponer el suyo y seguir el proceso mediante el cual se adoptaba la decisión. La cuestión, como nos planteaba la TRA, es considerar que, junto a un entorno deliberativo, el individuo puede modificar sus preferencias o aceptar, motivado racionalmente, una decisión a priori contraria o distinta a la suya.

\section{Conclusión}

En este artículo se ha planteado un acercamiento a la teoría democrática desde la teoría de la decisión. Se ha discutido, en primer lugar, sobre las implicaciones de pensar en la idea de la representación de las preferencias de los ciudadanos en democracia según consideremos éstas formadas y definidas o adaptativas y ajustables a la naturaleza del entorno político. Hemos considerado que la concepción o la interpretación dada a la formación y naturaleza de las preferencias implica una determinada concepción sobre la forma de representación de las mismas. Así, dado el carácter formado y predefinido que la teoría de la elección racional atribuye a las preferencias individuales, la forma apropiada de representarlas será considerando a todas ellas iguales y manteniendo su formación y desarrollo fuera del escenario político. Por su parte, si pensamos en las preferencias como opiniones y deseos adaptables, tanto al entorno como a la interacción con otros agentes, es posible pensar en otros mecanismos de representación más implicativos políticamente, tales como la deliberación.

Llegados a este punto, en este trabajo, nos hemos preguntado por la posibilidad misma de entender de forma adaptativa las preferencias individuales de los ciudadanos. La respuesta a esta cuestión ha venido de la mano de la teoría de la racionalidad acotada. La principal novedad de esta teoría es su definición de la racionalidad individual sobre la base de la relación entre individuo y entorno o, dicho con otras palabras, mediante el reconocimiento de que la racionalidad de los agentes reales queda definida a través del ajuste adaptati- 
vo entre los objetivos del agente y las características del contexto de decisión. En definitiva, el principal argumento sobre el que descansan los supuestos fundamentales de la teoría de la racionalidad acotada es el reconocimiento de la importancia de las restricciones del entorno de decisión a la hora de definir las preferencias de los agentes racionales.

Por último, en la tercera parte de este trabajo, nos hemos preguntado, dada la centralidad del entorno de decisión en la generación y formación de las preferencias individuales, por la posibilidad de una participación deliberativa. Consideramos que, dada la preeminencia del entorno, la cuestión principal se centra en si los procedimientos y las reglas deliberativas hacen posible un escenario en el que los ciudadanos puedan definir y discutir sus opiniones y preferencias. Así, hemos narrado los procedimientos, como también los resultados, de algunas experiencias deliberativas con la vista puesta en mostrar la posibilidad práctica de este tipo de experiencias, así como los criterios para generar y garantizar la deliberación. En nuestra opinión, si consideramos la adaptabilidad de las preferencias de la forma descrita por la TRA y garantizamos las condiciones de participación deliberativa, podemos concluir que es posible pensar en la representación deliberativa de las preferencias de los ciudadanos.

\section{Bibliografía}

ARROW, K. (1974). Elección social y valores individuales. Madrid: Ministerio de Hacienda. Bentham, J. (1996). An Introduction to the Principles Morals and Legislation. Oxford: Oxford University Press.

Bohman, J. (1998). "The Coming of Age of Deliberative Democracy». Journal of Political Philosophy, no 6, p. 400-425.

Cohen, J. (1986). «An Epistemic Conception of Democracy». Ethics, no 97, p. 26-38.

- (1998). «Democracy and liberty». En: ELSTER, John (ed.). Deliberative democracy. Cambridge University Press, p. 185-231.

Comité de Ministros del Consejo de Europa (2001). Recomendación (2001).

19: «La participación de los ciudadanos en la vida pública local», ed. Fundació Carles Pi i Sunyer d'Estudis Autonòmics i Locals, 2002. Puede consultarse en www.pisunyer.org.

Craven, J. (1992). Social choice: a frame work for collective decisions and individual judgments. Cambridge: Cambridge University Press.

DAHL, R. A. (1989). Democracy and its critics. New Haven.

- (1998). On democracy. Yale University Press.

ELSTER, J. (1988). Uvas amargas. Barcelona: Península.

- (1986). Rational choise: Readings in social and political theory. Oxford: Basil Blackwell. FonT, J. (coord.) (2001). Ciudadanos y decisiones públicas. Barcelona: Ariel.

FreY, B. S.; KUCHER, M.; STUTZER, A. (2001). «Outcome, process and power in direct democracies». New econometric results, public choice, $\mathrm{n}^{\circ} 107$ (3-4), p. 271-293. Friedman, M. (1953). Essays in positive economics. Chicago: Chicago University Press. GANUZA, E. (2005). «Democracia y nuevos horizontes: emergencia y límites de los Presupuestos Participativos en España». Acciones e Investigaciones Sociales, marzo, p. 3-37. 
- (2004). «La participación ciudadana en el ámbito local europeo». Revista de Estudios Europeos, no 38, septiembre-diciembre, p. 77-92.

Gargarella, R. (1998). «Full representation, deliberation and imparciality». En: ElSTER, John (ed.). Deliberative democracy. Cambridge University Press, p. 260-280.

Gigerenzer, G. (2000). Adaptative thinking. Rationality in the real word. Oxford: Oxford University Press.

Gigerenzer, G.; SELTEN, R. (2002). Bounded rationality. The adaptative toolbox. Cambridge Mass: The MIT Press.

Habermas, J. (1988). La teoría de la acción comunicativa. Madrid: Taurus.

- (2000). Facticidad y validez. Sobre el derecho y el Estado democrático de derecho en términos de teoría del discurso. Madrid: Trotta.

Hardin, R. (1995). One for All. The Logic of Group Conflict. Princenton: Princenton University Press.

Hume, D. (1988). Tratado de la naturaleza humana. Madrid: Tecnos.

KYMLICKA, W. (2003). La política vernácula: nacionalismo, multiculturalismo y ciudadanía. Barcelona: Paidos.

LitTle, I. M. D. (1950). A critique of welfare economics. Oxford: Clarendon Press.

MiLl, J. S. (1971). El utilitarismo. Barcelona: Orbis.

MOUfFE, C. (1993). The return of the political. Londres: Verso.

Nicholson, W. (2002). Teoría microeconómica: Principios básicos y ampliaciones. Madrid: Thomson.

OCDE (2001). Citizens as partners: Information, consultation and public participation in policy-making. París: OCDE.

PNUD (2002). Informe sobre desarrollo humano: profundizar la democracia en un mundo fragmentado. Madrid: Mundi-Prensa.

PRZEWORSKI, A. (1998). «Deliberation and ideological domination». En: ELSTER, John (ed.). Deliberative Democracy. Cambridge University Press, p. 140-160.

Putnam, R. (1993). Making democracy work. Princeton: Princeton University Press. RAWLS, J. (1971). Teoría de la justicia. Madrid: FCE.

RESCHER, N. (1993). La racionalidad: Una indagación filosófica sobre la naturaleza y la justificación de la razón. Madrid: Tecnos.

RoBles, J. M. (2005). "Racionalidad acotada: heurísticos y acción individual». Theoria, $14(1)$, p. 37-46.

SAMPEDRO, V. (2000). Opinión pública y democracia deliberativa. Madrid: Istmo.

SAmuelson, P. A. (1947). Foundations of economic analysis. Cambridge: Harvard University Press.

SARTORI, G. (1999). "En defensa de la representación política». Claves, no 91, abril, p. $2-7$.

- (1988). Teoría de la democracia. Madrid: Alianza.

SCHUMPETER, Joseph A. (1984). Capitalismo, socialismo y democracia. Barcelona: Folio.

SELTEN, R. (1998). "Aspiration Adaptation Theory». Journal of Mathematical Psychology, no 42, p. 191-214.

SEN, A. K. (1986). «Behaviour and the Concept of Preference». En: ELSTER, J. (1986). Rational choise. Readings in social and political theory. Oxford: Basil Blackwell.

SEN, A. (2000). Development as freedom. Nueva York: Anchor Books.

SimON, H. (1983). Reason in human affairs. Oxford: Basil Blackwell.

SinTOMER, Y. (2004). "Los presupuestos participativos en Europa: retos y desafíos».

Ponencia presentada en el IX Congreso CLAD sobre la Reforma del Estado y de la Administración Pública, Madrid, del 2 al 5 de noviembre. Mimeo. 
SubIRATS, J. (2003). Democracia representativa y democracia participativa ¿Dilema o complemento? Barcelona: Attac-Catalunya.

Tversky, A.; Kahneman, D. (eds.) (2000). Choices, values and frames. Cambridge: Cambridge University Press.

TodD, P.; GigerenZER, G. (2003). «Bounding rationality to the world». Journal of Economic Psychology, vol. 24, no 2, p. 143-165.

Weber, M. (1991). Escritos políticos. Madrid: Alianza. 